\title{
Papel de la cirugía en la enfermedad degenerativa espinal. Análisis de revisiones sistemáticas sobre tratamientos quirúrgicos y conservadores desde el punto de vista
} de la medicina basada en la evidencia

\author{
P.D. Delgado-López; A. Rodríguez-Salazar; J.M. Castilla-Díez; V. Martín-Velasco y O. Fernández-Arconada
}

Servicio de Neurocirugía. Hospital General Yagüe. Avda. del Cid 96. 09005 Burgos.

\section{Resumen}

Introducción. Alrededor del $\mathbf{7 0 - 8 0 \%}$ de la población presentará dolor de espalda incapacitante algún momento en su vida como consecuencia de la Enfermedad Degenerativa Espinal (EDE). Los costes globales que genera la enfermedad se estiman en torno al $1-2 \%$ del PIB anualmente. Desde el punto de vista de la Medicina Basada en la Evidencia (MBE), se constata una llamativa discrepancia entre la enorme disponibilidad y creciente uso de técnicas quirúrgicas (en especial de fusión espinal) y la escasa evidencia científica que apoya su utilización.

Material y métodos. Hemos revisado cuidadosamente todos los metaanálisis referentes a tratamientos de la EDE publicados hasta Diciembre de 2003 y hemos clasificado las recomendaciones terapéuticas en niveles de evidencia (fuerte, moderada, limitada o ausencia de evidencia), tanto para tratamientos quirúrgicos como conservadores, siguiendo las pautas de la MBE.

Resultados. Identificamos 44 metaanálisis de interés $(9$ sobre cirugía lumbar, 3 sobre cirugía cervical y 32 sobre otros tratamientos). Desde el punto de vista quirúrgico, sólo alcanza nivel de evidencia fuerte la laminectomía precoz en síndrome de cola de caballo por extrusión discal; la superioridad de la discectomía simple o microdiscectomía frente a quimionucleolisis en prolapso discal y espondilosis; y la cirugía de fusión (en principio, no instrumentada) en espondilolistesis ístmica del adulto o degenerativa asociada a estenosis lumbar. En espondilosis cervical con radiculo y/o mielopatía cervical leve, la discectomía más fusión no supera a la discectomía simple y ésta es dudosamente superior a la historia natural de la enfermedad más allá de 24 meses. La utilización profiláctica de antibióticos en cirugía espinal es beneficiosa. No se demuestra beneficio de la cirugía en dolor discogénico. Ninguna terapia conservadora alcanza el nivel de evidencia fuerte. Los antidepresivos mejoran la percepción del dolor pero no

Recibido: 18-08-04. Aceptado: 14-10-04 la funcionalidad.

Discusión. A pesar de que se ha doblado el porcentaje de cirugías de instrumentación lumbar en las últimas dos décadas y crece a un ritmo del $20 \%$ anual, no se ha demostrado de forma fehaciente una mejoría en los resultados clínicos ni siquiera en las tasas globales de artrodesis. Este llamativo incremento del uso de la cirugía en procesos diferentes a las deformidades espinales y espondilolistesis aisladas o acompañadas de estenosis del canal lumbar, quizá obedece a múltiples factores técnicos y clínico-epidemiológicos donde no podemos obviar la enorme trascendencia económica que subyace. Resulta crucial diferenciar qué subgrupos de pacientes con EDE se benefician claramente de la cirugía. Desde el punto de vista ético empieza a plantearse la necesidad de diseñar ensayos clínicos que incorporen placebos quirúrgicos, dada la escasa evidencia científica que apoya la cirugía espinal a día de hoy. La mayor parte de los tratamientos conservadores tienen una eficacia moderada o leve (casi siempre transitoria) y, probablemente, deban utilizarse en combinación.

Conclusiones. La cirugía de la EDE se asienta sobre pilares inseguros habida cuenta de que la mayor parte de las técnicas que se indican no están avaladas por recomendaciones de primera clase en términos de MBE. Parece necesario consensuar, desde las organizaciones que estudian la columna degenerativa, guías de práctica clínica en lo referente al tratamiento integral y multidisciplinado de la EDE, a sabiendas que, hasta hoy, pocos tratamientos alteran de forma positiva $y$ duradera la historia natural de la enfermedad.

PALABRAS CLAVE: Dolor lumbar crónico. Discopatía degenerativa. Fusión lumbar. Instrumentación espinal. Tratamientos conservadores.

Abreviaturas: EDE: enfermedad degenerativa espinal. MBE: medicina basada en la evidencia. PIB: producto interior bruto. SDD: spinal degenerative disease. 
Role of surgery in spinal degenerative disease. Analysis of systematic reviews on surgical and conservative treatments from an evidence-based approach

\section{Summary}

Introduction. The lifetime prevalence of invalidating back pain in general population caused by Spinal Degenerative Disease (SDD) is about $\mathbf{7 0 - 8 0 \%}$. Global costs related to this disease are enormous $(1-2 \%$ gross domestic product). From an Evidence-based point of view, there is a striking discrepancy between the use of many available surgical techniques (especially for spinal fusion) and the lack of scientific support.

Methods. The authors carefully reviewed all published metaanalysis on SDD therapies up to December 2003. Treatment recommendations were classified according to levels of evidence (strong, moderate, mild or lack of evidence) for both surgical and conservative measures.

Results. Forty-four metaanalysis were selected (nine on lumbar surgery, three on cervical surgery and thirty-two on other therapies). Relating surgery, there is strong evidence favouring early laminectomy in cauda equina syndrome secondary to lumbar disc herniation; discectomy or microdiscectomy are superior to chemonucleolysis in lumbar prolapse and spondylosis; and fusion surgery (probably noninstrumented) in adult isthmic spondylolysthesis or degenerative spondylolysthesis with spinal stenosis. In cervical spondylosis and radiculomyelopathy, discectomy seems as efective as discectomy plus fusion, which does not seem to be better than untreated SDD beyond 24 months. Preoperative antibiotics seem to prevent infection in spinal surgery. No benefit of surgery is demonstrated in discogenic pain. None of conservative therapies are supported by strong evidence. Antidepressants improve pain perception but do not influence the functional status.

Discussion. Although lumbar instrumented surgery has nearly doubled over two decades and the anual growth is about $20 \%$, clinical results do not seem to have improved, not even global fusion rates. The increasing use of fusion surgery for cases other than spinal deformities, spondylolysthesis or spinal stenosis plus lysthesis may be related to multiple technical and clinical-epidemiological factors where huge financial and commercial interests must be considered. It is crucial to differenciate subsets of patients prone to beneft from surgery. It is discussed whether randomized trials incorporating sham operations are ethically justifiable, because of the lack of sould evidence for many spinal procedures. The efficacy of most conservative treatments is mild or moderate (mainly transient) and they should be probably used in combination.

Conclusions. There is no strong evidence favouring most of surgical procedures for SDD from an evidencebased approach. It seems neccessary that scientific organizations studying SDD create clinical guidelines relating its multidisciplinary and integral management, recognizing that, up to now, few interventions positively modify in the long-term the natural history of the disease.

KEY WORDS: Low back pain. Degenerative disc disease. Lumbar fusion. Spinal instrumentation. Conservative treatments.

\section{Introducción}

La enfermedad degenerativa espinal (EDE) engloba un conjunto de patologías cuyo síntoma fundamental es el dolor cervical, dorsal o lumbar crónicos. Se considera la primera causa de baja laboral en los países industrializados y la segunda en motivo de consulta en atención primaria, sólo por detrás del resfriado común. Se estima que supone entre el 1-2\% del producto interior bruto de un país anualmente en gastos médicos y sociales; tres veces más que todo el dinero destinado al tratamiento de todas las formas de cáncer ${ }^{49}$. Aproximadamente entre el 70-80\% de la población sufre a lo largo de su vida algún episodio de dolor de espalda incapacitante. Sólo la cefalea es más prevalente que el dolor lumbar entre los calificados como dolores crónicos ${ }^{50}$.

La Medicina Basada en la Evidencia (MBE) aporta criterios modernos y sólidos relativos a la efectividad de los tratamientos médicos en función de la calidad de los estudios que los avalan. El conocimiento de la historia natural de las enfermedades es crucial para estimar la efectividad de dichos tratamientos. La literatura sobre EDE de la que se dispone en la actualidad es enorme, aunque muchas veces contradictoria. Una lectura crítica de la misma evidencia una llamativa discrepancia entre la enorme disponibilidad de técnicas quirúrgicas (en especial de fusión vertebral) y multitud de tratamientos conservadores, y la escasez y/o mala calidad de los estudios que defienden su uso. En los últimos veinte años se ha constatado un aumento progresivo en la aplicación de procedimientos quirúrgicos sobre la columna con indicaciones en constante aumento ${ }^{8,49}$. Esta tendencia resulta preocupante tanto por la falta de un soporte científico adecuado como por los ingentes costes que genera para la sociedad.

El propósito de este trabajo es reunir y resumir el conocimiento actual referente a los diversos tratamientos médicos y quirúrgicos de los que se dispone para manejar la enfermedad degenerativa espinal y el dolor crónico de la espalda, esencialmente el dolor lumbar, desde el punto de 
vista de la MBE.

\section{Material y métodos}

Hemos llevado a cabo una lectura cuidadosa de las revisiones sistemáticas (metaanálisis) publicadas hasta Diciembre de 2003 que hacen referencia específicamente a tratamientos de la EDE. Se realizó mediante búsqueda informatizada en la base de datos MedLine, en la dirección de Internet: www.ncbi.nim.nih.gov/PubMed. El proceso de búsqueda comprendió únicamente los trabajos indexados bajo la restricción de ser clasificados como metaanálisis. Se emplearon las siguientes palabras clave: Lumbar disc hemiation, lumbar stenosis, degenerative spine disease, lumbar spodylolisthesis, lumbar arthrodesis, lumbar arthrosis, nucleotomy, spinal fusion, spinal instrumentation, spinal surgery, neuroreflexotherapy, chronic back pain, low back pain, neck pain y acupuncture.

De todas las entradas obtenidas se seleccionaron sólo aquellos estudios con implicaciones terapéuticas, en principio, en cualquier idioma. Posteriormente se obtuvo copia escrita de cada trabajo para su estudio y análisis. En los casos en los que el estudio estuvo escrito en idiomas diferentes a castellano, italiano, inglés o francés o no se pudo disponer de la publicación, únicamente se empleó la información contenida en el resumen.

Según las guías propuestas por la metodología de la $\mathrm{MBE}^{49}$, se definen como Evidencia Fuerte (de clase A o de tipo 1) aquella refrendada por metaanálisis, estudios multicéntricos, aleatorizados, prospectivos y ciegos; Evidencia Moderada (de clase B o tipo 2) aquella refrendada por estudios similares a los previos pero sin aleatorización o por estudios observacionales de buena calidad; y Evidencia Limitada o Leve (de clase C o tipo 3) aquella refrendada por estudios no prospectivos, cohortes, casos y controles, series de casos y opiniones de expertos. Cualquier otro tipo de estudio no se considera que aporte evidencia de clase alguna (Evidencia de clase D). Ver Tabla de Niveles de Evidencia $^{49}$ (Tabla 1).
Las revisiones realizadas por la Colaboración Cochrane se ciñen estrictamente a la metodología anteriormente expuesta, lo que no siempre ocurre en muchos de los estudios que revisamos. Algunos trabajos con fecha de publicación posterior a Diciembre de 2003 no se incluyen en el grupo de análisis, pero fueron tenidos en cuenta en la discusión por el gran interés de su contenido ${ }^{8,15}$.

\section{Resultados}

Tras la introducción de las palabras clave en MedLine, y una depuración inicial, se obtuvieron 62 trabajos de tipo metaanálisis (14 referentes a cirugía de la columna y 48 sobre otro tipo de tratamientos). Una revisión cuidadosa de los resúmenes descartó 18 trabajos que no trataban específicamente sobre tratamientos de la EDE. De los 44 estudios resultantes ( 9 de cirugía de la columna lumbar, 3 de tratamientos sobre la columna cervical y 32 sobre otros tratamientos), en 10 no se pudo obtener original del trabajo en copia papel ${ }^{3,9,11,29,37,47,58,67,72,75}$ ( 5 se encontraban en idiomas distintos a los referidos ${ }^{29,47,58,72,75}$ ), por lo que se emplearon únicamente los datos del resumen.

La Tabla de Resultados de Estudios (Tabla 2) resume las características, resultados y conclusiones relevantes de cada trabajo, y se comentan las limitaciones y calidad de los mismos.

La Tabla de Recomendaciones según MBE (Tabla 3) reúne las conclusiones que se extraen de los estudios revisados y su nivel de evidencia. A continuación se describen de forma resumida bajo dos epígrafes: tratamientos quirúrgicos y tratamientos no quirúrgicos.

\section{Tratamientos quirúrgicos}

Desde el punto de vista quirúrgico existen muy pocas recomendaciones avaladas por estudios de clase A relativos a la EDE. Los pacientes con hernia discal lumbar y radiculopatía se benefician de forma similar tanto de la discectomía simple como de la discectomía más fusión ${ }^{32,25}$

Tabla 1

Tabla de Niveles de Evidencia

\begin{tabular}{|c|l|l|}
\hline Clase & Denominación & Estudios \\
\hline A & FUERTE & Metaanálisis o revisión sistematizada de alta calidad de dos o más estudios. \\
\hline B & MODERADA & Uno o más RCTs o estudios observacionales de alta calidad. \\
\hline C & LIMITADA & Evidencia insuficiente o no concluyente (ningún RCT o RCT u observacional pobres) \\
\hline D & NO EVIDENCIA & Sin base científica aceptable \\
\hline
\end{tabular}


Tabla 2

Tabla de resultados de estudios tipo metaanálisis

\begin{tabular}{|c|c|c|c|c|}
\hline Autor y año & Material analizado & Resultados y conclusiones & Limitaciones & Tipo de Estudio \\
\hline $\begin{array}{l}\text { Anh UM et al, } \\
2000^{1}\end{array}$ & $\begin{array}{l}42 \text { estudios, } \\
322 \text { pacientes }\end{array}$ & $\begin{array}{l}\text { Mejor recuperación motora, sensitiva y } \\
\text { esfínteres en intervenidos antes de } 48 \mathrm{~h} \text { de } \\
\text { síndrome de cola de caballo por hernia discal }\end{array}$ & & Metaanálisis \\
\hline $\begin{array}{l}\text { Gibson JN et } \\
\text { al, } 1999^{25}\end{array}$ & $\begin{array}{l}26 \text { RCTs sobre } \\
\text { prolapso y } 14 \\
\text { sobre espondilosis }\end{array}$ & $\begin{array}{l}\text { En prolapso y espondilosis lumbar, } \\
\text { discectomía es similar a microdiscectomía } \\
\text { que es superior a quimionucleolisis y a } \\
\text { placebo. No hay evidencia a favor de } \\
\text { ninguna clase de descompresión o fusión } \\
\text { en espondilosis degenerativa. La } \\
\text { instrumentación facilita la fusión pero no } \\
\text { tiene repercusión clínica }\end{array}$ & $\begin{array}{l}\text { Sólo un estudio } \\
\text { compara } \\
\text { discectomía con } \\
\text { tratamiento } \\
\text { conservador }\end{array}$ & $\begin{array}{l}\text { Revisión de la } \\
\text { Colaboración } \\
\text { Cochrane. } \\
\text { Metaanálisis }\end{array}$ \\
\hline $\begin{array}{l}\text { Niggemeyer O } \\
\text { et al, } 1997^{52}\end{array}$ & $\begin{array}{l}30 \text { estudios, } \\
1668 \text { pacientes }\end{array}$ & $\begin{array}{l}\text { En estenosis lumbar degenerativa de } \\
<8 \text { años de evolución lo mejor es } \\
\text { descompresión sin fusión. } \mathrm{Si}>15 \text { años: } \\
\text { descompresión + fusión instrumentada. } \\
\text { Globalmente la descompresión sola tiene } \\
\text { mejor resultado y menos morbilidad }\end{array}$ & $\begin{array}{l}\text { Estudios } \\
\text { publicados } \\
\text { hasta } 1995\end{array}$ & Metaanálisis \\
\hline $\begin{array}{l}\text { Turner JA et } \\
\text { al, } 1992^{63}\end{array}$ & 74 estudios & $\begin{array}{l}\text { Resultados buenos o excelentes en el } 64 \% \\
\text { de los intervenidos de estenosis lumbar } \\
\text { degenerativa }\end{array}$ & $\begin{array}{l}\text { Estudios } \\
\text { publicados hasta } \\
\text { 1992. Defectos de } \\
\text { diseño y análisis }\end{array}$ & $\begin{array}{l}\text { Metanaálisis. } \\
\text { Evidencia } \\
\text { dudosa }\end{array}$ \\
\hline $\begin{array}{l}\text { Burkus JK et } \\
\text { al, } 2003^{11}\end{array}$ & $\begin{array}{l}679 \text { pacientes, } \\
277 \text { rhBMP-2 y } \\
402 \text { cresta ilíaca }\end{array}$ & $\begin{array}{l}\text { En artrodesis lumbar anterior, la utilización } \\
\text { de caja rellena de rhBMP-2 frente a cresta } \\
\text { ilíaca, mejora el resultado según escala } \\
\text { Oswestry hasta } 24 \text { meses, con menos } \\
\text { morbilidad y estancia, más tasa de fusión y } \\
\text { vuelta antes al trabajo }\end{array}$ & $\begin{array}{l}\text { Heterogeneidad } \\
\text { de pacientes } \\
\text { y diagnósticos }\end{array}$ & $\begin{array}{l}\text { Metaanálisis. } \\
\text { Análisis de } \\
\text { covariancia }\end{array}$ \\
\hline $\begin{array}{l}\text { Schmid, } \\
2000^{58}\end{array}$ & $\begin{array}{l}69 \text { estudios: } \\
22567 \\
\text { pacientes }\end{array}$ & $\begin{array}{l}\text { Microdiscectomía supera a discectomía } \\
\text { estándar en prolapso o HDL. Ambas mejor } \\
\text { que cualquier técnica percutánea. } \\
\text { Recidivas: } 4 \% \text { en las discectomías, } 14 \% \text { en } \\
\text { nucleotomía endoscópica y } 18 \% \text { en } \\
\text { quimionucleolisis o laser }\end{array}$ & $\begin{array}{l}\text { Estudios hasta } \\
\text { año } 2000 . \\
\text { Artículo en } \\
\text { alemán. Utiliza } \\
\text { algunos trabajos } \\
\text { retrospectivos }\end{array}$ & Metaanálisis \\
\hline $\begin{array}{l}\text { Knight MT et } \\
\text { al, } 2001^{37}\end{array}$ & $\begin{array}{l}716 \text { pacientes, } \\
958 \\
\text { procedimientos }\end{array}$ & $\begin{array}{l}\text { En dolor lumbar crónico y ciática, la } \\
\text { foraminoplastia endoscópica por laser } \\
\text { tiene menos complicaciones }(1.6 \%) \text { a } 6 \\
\text { semanas que las técnicas abiertas }(6-12 \%)\end{array}$ & $\begin{array}{l}\text { Realizado en un } \\
\text { solo centro en } \\
\text { Reino Unido. No } \\
\text { estudia resultados }\end{array}$ & $\begin{array}{l}\text { Compara sus } \\
\text { resultados } \\
\text { contra } \\
\text { metaanálisis }\end{array}$ \\
\hline $\begin{array}{l}\text { Barker FG2nd, } \\
2002^{6}\end{array}$ & $\begin{array}{l}6 \mathrm{RCTs}, 843 \\
\text { pacientes }\end{array}$ & $\begin{array}{l}\text { Los antibióticos profilácticos en cirugía } \\
\text { espinal reducen a un tercio la tasa de } \\
\text { infecciones. Se mantiene el efecto aun en } \\
\text { baja tasa previa, con independencia de } \\
\text { número de dosis y cobertura añadida a } \\
\text { gram negativos aparte de gram positivos. }\end{array}$ & $\begin{array}{l}\text { Cuatro estudios } \\
\text { de neurocirugía } \\
\text { general, } 1 \text { de } \\
\text { cirugía espinal } \\
\text { y } 1 \text { de cirugía } \\
\text { ortopédica }\end{array}$ & $\begin{array}{l}\text { Metaanálisis. } \\
\text { Regresión } \\
\text { bayesiana }\end{array}$ \\
\hline $\begin{array}{l}\text { Ahn UN et al, } \\
2001^{2}\end{array}$ & 4 estudios & $\begin{array}{l}\text { En síndrome de cauda equina por } \\
\text { espondilitis anquilosante, los AINEs } \\
\text { mejoran el dolor pero no el déficit y la } \\
\text { cirugía de la "ectasia dural" lumbosacra por } \\
\text { derivación lumboperitoneal o laminectomía } \\
\text { mejora o detiene la progresión del déficit. }\end{array}$ & & Metaanálisis \\
\hline
\end{tabular}




\begin{tabular}{|c|c|c|c|c|}
\hline $\begin{array}{l}\text { Floyd T et al, } \\
2000^{20}\end{array}$ & $\begin{array}{l}4 \text { estudios, } \\
310 \text { pacientes } \\
379 \text { niveles }\end{array}$ & $\begin{array}{l}\text { En radiculopatía cervical espondilótica, el } \\
\text { autoinjerto en ACDF produce mayor tasa } \\
\text { de fusión radiológica y menos colapso pero } \\
\text { no mejor resultado clínico. Considerar la } \\
\text { morbilidad del lugar del injerto autólogo }\end{array}$ & $\begin{array}{l}\text { No demuestra } \\
\text { beneficio clínico } \\
\text { a favor del } \\
\text { autoinjerto }\end{array}$ & Metaanálisis \\
\hline $\begin{array}{l}\text { Fouyas IP et } \\
\mathrm{al}, 2002^{21}\end{array}$ & $\begin{array}{l}2 \text { RCTs, } \\
130 \text { pacientes }\end{array}$ & $\begin{array}{l}\text { En radiculopatía o mielopatía cervical } \\
\text { espondilótica la cirugía de descompresión } \\
\text { es superior a la fisioterapia o collarín a } \\
\text { corto plazo pero se iguala a } 1 \text { año } \\
\text { (radiculopatía)y a } 2 \text { años (mielopatía leve) }\end{array}$ & $\begin{array}{l}\text { Tratamientos } \\
\text { quirúrgicos } \\
\text { diversos } \\
\text { (descompresión } \\
\text { con o sin fusión) }\end{array}$ & $\begin{array}{l}\text { Revisión de la } \\
\text { Colaboración } \\
\text { Cochrane }\end{array}$ \\
\hline $\begin{array}{l}\text { Gross AR et } \\
\text { al, } 1996^{28}\end{array}$ & $\begin{array}{l}24 \text { RCTs y } 8 \\
\text { estudios } \\
\text { diversos }\end{array}$ & $\begin{array}{l}\text { Evidencia moderada a favor de } \\
\text { tratamientos físioterápicos en dolor cervical } \\
\text { mecánico del adulto. Evidencia leve a favor } \\
\text { de terapia electromagnética y en contra de } \\
\text { laserterapia }\end{array}$ & $\begin{array}{l}\text { Estudios } \\
\text { hasta } 1993\end{array}$ & Metaanálisis \\
\hline $\begin{array}{l}\text { Akai et al, } \\
2002^{3}\end{array}$ & 5 RCTs & $\begin{array}{l}\text { Evidencia estadística de que la } \\
\text { estimulación eléctrica o electromagnética } \\
\text { favorece la fusión espinal }\end{array}$ & $\begin{array}{l}\text { Baja calidad de } \\
\text { los estudios }\end{array}$ & Metaanálisis \\
\hline $\begin{array}{l}\text { Van Buyten JP } \\
\text { et al, 200166 }\end{array}$ & 217 pacientes & $\begin{array}{l}\text { En lumbalgia crónica, la estimulación } \\
\text { epidural produce un } 68 \% \text { de resultados } \\
\text { subjetivos buenos o excelentes a } 4 \text { años y } \\
\text { vuelven al trabajoo un } 31 \%\end{array}$ & $\begin{array}{l}\text { Un solo centro de } \\
\text { Dolor en Bélgica }\end{array}$ & $\begin{array}{l}\text { Cohorte } \\
\text { prospectiva } \\
\text { seguida } \\
10 \text { años }\end{array}$ \\
\hline $\begin{array}{l}\text { Turner JA et } \\
\text { al, } 1995^{65}\end{array}$ & 39 estudios & $\begin{array}{l}\text { La estimulación epidural en Failed-Back } \\
\text { syndrome produce un alivio del dolor en } \\
59 \% \text { pacientes. Tuvieron complicaciones } \\
\text { menores en el } 42 \% \text { de los casos }\end{array}$ & $\begin{array}{l}\text { Estudios hasta } \\
\text { 1994. Seguimiento } \\
\text { mediano de sólo } \\
16 \text { meses }\end{array}$ & $\begin{array}{l}\text { Metaanálisis } \\
\text { de series } \\
\text { de casos }\end{array}$ \\
\hline $\begin{array}{l}\text { Kovacs FM et } \\
\text { al, } 2002^{41}\end{array}$ & $\begin{array}{l}1 \mathrm{RCT}, 104 \\
\text { pacientes }(45 \\
\text { controles) }\end{array}$ & $\begin{array}{l}\text { La neurorreflejoterapia añadida al } \\
\text { tratamiento estándar en lumbalgia crónica } \\
\text { produce menos dolor en VAS, dolor } \\
\text { referido, score en Roland-Morris, menos } \\
\text { consulta con especialista y menos gasto en } \\
\text { Rx y medicamentos para el dolor }\end{array}$ & $\begin{array}{l}\text { Asignación } \\
\text { aleatoria de } \\
\text { médicos, no de } \\
\text { pacientes. } \\
\text { Seguimiento } \\
\text { a } 365 \text { días }\end{array}$ & $\begin{array}{l}\text { Ensayo } \\
\text { clínico } \\
\text { aleatorizado } \\
\text { sin ciego }\end{array}$ \\
\hline $\begin{array}{l}\text { Kovacs FM et } \\
\text { al, } 1997^{40}\end{array}$ & $\begin{array}{l}1 \mathrm{RCT}, \\
78 \text { pacientes } \\
(37 \text { controles })\end{array}$ & $\begin{array}{l}\text { La neurorreflejoterapia (una sesión) en } \\
\text { lumbalgia crónica frente al procedimiento } \\
\text { hecho sin efecto (sham) alivia } \\
\text { inmediatamente el dolor en mayor } \\
\text { porcentaje que en controles, hasta } 45 \text { días }\end{array}$ & $\begin{array}{l}\text { Pacientes } \\
\text { reclutados por } \\
\text { rehabilitadores y } \\
\text { reumatólogos en } \\
\text { tres centros } \\
\text { de Madrid }\end{array}$ & $\begin{array}{l}\text { Ensayo } \\
\text { clínico } \\
\text { aleatorizado } \\
\text { ciego y } \\
\text { controlado }\end{array}$ \\
\hline $\begin{array}{l}\text { Smith LA et } \\
\text { al, } 2000^{60}\end{array}$ & $13 \mathrm{RCTs}$ & $\begin{array}{l}\text { La acupuntura frente a placebo en dolor } \\
\text { lumbar o cervical crónicos es efectiva } \\
\text { (5RCTs) o no es efectiva ( } 8 \text { RCTs, con } \\
\text { mayor puntuación en Oxford Pain } \\
\text { Validity Scale) }\end{array}$ & $\begin{array}{l}\text { Los estudios de } \\
\text { mejor calidad } \\
\text { fueron negativos. } \\
\text { Evidencia no } \\
\text { concluyente }\end{array}$ & Metaanálisis \\
\hline $\begin{array}{l}\text { Van Tudler } \\
\text { MW et al, } \\
1999^{70}\end{array}$ & $\begin{array}{l}11 \text { RCTs, } \\
\text { sólo } 2 \text { de } \\
\text { alta calidad }\end{array}$ & $\begin{array}{l}\text { No hay evidencia de que la acupuntura en } \\
\text { lumbalgia crónica tenga más efecto que no } \\
\text { tratar. Evidencia moderada de que no sea } \\
\text { más efectiva que TENS o inyecciones en } \\
\text { trigger-points; y limitada evidencia de que } \\
\text { no sea más efectiva que placebo o sham }\end{array}$ & $\begin{array}{l}\text { Revisión sólo } \\
\text { cualitativa } \\
\text { debido a la } \\
\text { heterogeneidad } \\
\text { de los datos }\end{array}$ & $\begin{array}{l}\text { Siguen pautas } \\
\text { de la } \\
\text { Colaboración } \\
\text { Cochrane. } \\
\text { Metaanálisis }\end{array}$ \\
\hline $\begin{array}{l}\text { Ernst E et } \\
\text { al, } 1998^{16}\end{array}$ & $12 \mathrm{RCTs}$ & $\begin{array}{l}\text { La acupuntura es superior a otros } \\
\text { tratamientos controles en lumbalgia } \\
\text { crónica pero no hay evidencia de que } \\
\text { sea superior a placebo }\end{array}$ & $\begin{array}{l}\text { Sólo } 9 \text { RCTs } \\
\text { aportan datos útiles } \\
\text { para el análisis y } \\
\text { cálculo de OR }\end{array}$ & Metaanálisis \\
\hline
\end{tabular}




\begin{tabular}{|c|c|c|c|c|}
\hline $\begin{array}{l}\text { Assendelft WJ } \\
\text { et al, 2003 }\end{array}$ & 39 RCTs & $\begin{array}{l}\text { En lumbalgia aguda o crónica, los } \\
\text { tratamientos de Manipulación Espinal son } \\
\text { superiores sólo al sham e igual a } \\
\text { analgésicos, ejercicios físicos o escuela de } \\
\text { espalda. Independiente de la profesión del } \\
\text { manipulador y calidad del estudio }\end{array}$ & $\begin{array}{l}\text { Analiza pacientes } \\
\text { con seguimientos } \\
\text { cortos y largos y } \\
\text { con dolores agudos } \\
\text { y crónicos }\end{array}$ & $\begin{array}{l}\text { Cochrane Back } \\
\text { Review Group. } \\
\text { Metaanálisis. } \\
\text { Metarregresión }\end{array}$ \\
\hline $\begin{array}{l}\text { Brosseau L et } \\
\text { al, } 2002^{10}\end{array}$ & $\begin{array}{l}5 \text { RCTs, } 391 \\
\text { pacientes }(170 \\
\text { controles) }\end{array}$ & $\begin{array}{l}\text { El TENS aislado en lumbalgia crónica no es } \\
\text { superior al sham-TENS. Igual de inefectivo } \\
\text { el TENS convencional que el TENS } \\
\text { Acupuncture-like }\end{array}$ & $\begin{array}{l}\text { No tiene en cuenta } \\
\text { características y } \\
\text { duración del } \\
\text { tratamiento }\end{array}$ & Metaanálisis \\
\hline $\begin{array}{l}\text { Salerno SM et } \\
\text { al, } 2002^{56}\end{array}$ & $\begin{array}{l}9 \mathrm{RCTs} \text {, } \\
504 \text { pacientes }\end{array}$ & $\begin{array}{l}\text { Los antidepresivos en lumbalgia crónica } \\
\text { mejoran la intensidad del dolor frente a } \\
\text { placebo pero no las actividades diarias del } \\
\text { paciente. Tienen más efectos } 2^{\circ} \text { que el } \\
\text { placebo }\end{array}$ & $\begin{array}{l}\text { Sólo considera } \\
\text { literatura en inglés. } \\
\text { Siete brazos de } \\
\text { tratamiento sobre } \\
\text { depresión mayor }\end{array}$ & Metaanálisis \\
\hline $\begin{array}{l}\text { Goldberg MS } \\
\text { et al, } 2000^{26}\end{array}$ & 34 estudios & $\begin{array}{l}\text { Asociación positiva entre tabaco e } \\
\text { incidencia y prevalencia de lumbalgia } \\
\text { crónica (no ciática) en } 18 / 26 \text { estudios en } \\
\text { varones y } 18 / 20 \text { en mujeres }\end{array}$ & $\begin{array}{l}\text { Mayoría estudios } \\
\text { transversales. } \\
\text { Evidencia dudosa } \\
\text { debido a sesgos }\end{array}$ & $\begin{array}{l}\text { Metaanálisis } \\
\text { cualitativo }\end{array}$ \\
\hline $\begin{array}{l}\text { Assendelft WJ } \\
\text { et al, } 1996^{4}\end{array}$ & $\begin{array}{l}18 \text { RCTs, } \\
\text { baja calidad }\end{array}$ & $\begin{array}{l}\text { La quiropráctica en lumbalgia crónica o } \\
\text { aguda no parece ser efectiva, desde un } \\
\text { punto de vista cualitativo }\end{array}$ & $\begin{array}{l}\text { Análisis cualitativo } \\
\text { únicamente. } \\
\text { Estudios hasta } 1995\end{array}$ & $\begin{array}{l}\text { Metaanálisis } \\
\text { cualitativo }\end{array}$ \\
\hline $\begin{array}{l}\text { Koes BW } \\
\text { et al, } 1995^{38}\end{array}$ & $\begin{array}{l}12 \text { RCTs, } \\
8 \text { RCTs } \\
\text { buena } \\
\text { calidad }\end{array}$ & $\begin{array}{l}\text { La inyección epidural de esteroides es más } \\
\text { efectiva en lumbalgialciática que el } \\
\text { tratamiento de referencia en } 6 \text { estudios y } \\
\text { menos en otros } 6 \text { estudios. Beneficio } \\
\text { dudoso y en todo caso de corta duración }\end{array}$ & $\begin{array}{l}\text { De los } 4 \text { mejores } \\
\text { estudios, } \\
\text { evidencia } \\
\text { inconclusa }\end{array}$ & Metaanálisis \\
\hline $\begin{array}{l}\text { Niemisto L } \\
\text { et al, } 2003^{51}\end{array}$ & $\begin{array}{l}7 \text { RCTs, } \\
275 \\
\text { pacientes } \\
(134 \\
\text { controles })\end{array}$ & $\begin{array}{l}\text { La denervación por radiofrecuencia en } \\
\text { dolor discogénico no es efectiva (evidencia } \\
\text { limitada), en dolor cervical obtiene mejoría } \\
\text { a corto plazo sólo (evidencia limitada) y en } \\
\text { síndrome facetario lumbar es dudoso }\end{array}$ & $\begin{array}{l}6 \text { RCTs } \\
\text { de alta } \\
\text { calidad }\end{array}$ & $\begin{array}{l}\text { Revisión de } \\
\text { Colaboración } \\
\text { Cochrane. } \\
\text { Metaanálisis }\end{array}$ \\
\hline $\begin{array}{l}\text { Rohling ML } \\
\text { et al, } 1995^{55}\end{array}$ & $\begin{array}{l}32 \text { estudios, } \\
7651 \text { pacientes } \\
(3849 \\
\text { controles })\end{array}$ & $\begin{array}{l}\text { La compensación económica se relaciona } \\
\text { con mayor dolor referido por el paciente y } \\
\text { menor eficacia del tratamiento en dolor } \\
\text { crónico }\end{array}$ & $\begin{array}{l}72 \% \text { estudios } \\
\text { sobre lumbalgia } \\
\text { crónica } \\
\text { específicamente }\end{array}$ & Metaanálisis \\
\hline $\begin{array}{l}\text { Koes BW et } \\
\text { al, 1994 }\end{array}$ & 16 RCTs. & $\begin{array}{l}\text { La escuela de espalda parece tener efecto } \\
\text { positivo, especialmente la variante Swedish } \\
\text { Back-School intensiva } 3-5 \text { semanas, pero } \\
\text { de corta duración ( } 7 \text { RCTs) o eficacia } \\
\text { dudosa o nula (9 RCTs) }\end{array}$ & $\begin{array}{l}\text { Datos hasta } 1994 . \\
\text { Sólo } 2 \text { estudios de } \\
\text { calidad moderada }\end{array}$ & Metaanálisis \\
\hline $\begin{array}{l}\text { Hartvijsen J } \\
\text { et al, } 2002^{29}\end{array}$ & 35 estudios & $\begin{array}{l}\text { No parece existir asociación positiva entre } \\
\text { postura sentada en el trabajo u ocupación } \\
\text { sedentaria y lumbalgia. }\end{array}$ & $\begin{array}{l}\text { Estudio en Danés. } \\
\text { Sólo } 8 \text { estudios } \\
\text { de calidad }\end{array}$ & Metaanálisis \\
\hline $\begin{array}{l}\text { Van Poppel } \\
\text { MN et al, } \\
2000^{69}\end{array}$ & 33 estudios. & $\begin{array}{l}\text { Los soportes lumbares disminuyen la } \\
\text { flexoextensión y torsión lateral del tronco } \\
\text { (no la rotación) }\end{array}$ & $\begin{array}{l}\text { No aporta datos de } \\
\text { efectividad clínica }\end{array}$ & Metaanálisis \\
\hline $\begin{array}{l}\text { Bovenzi M } \\
\text { et al, } 1999^{9}\end{array}$ & $\begin{array}{l}17 \text { estudios, } \\
\text { ningún RCT. }\end{array}$ & $\begin{array}{l}\text { Asociación positiva entre exposición a } \\
\text { vibraciones de todo el cuerpo (laboral o no) } \\
\text { y más lumbalgia, ciática y cambios } \\
\text { degenerativos espinales. Relevancia clínica } \\
\text { dudosa por la calidad de los estudios. }\end{array}$ & $\begin{array}{l}\text { Mayoría de los } \\
\text { estudios son de } \\
\text { tipo transversal y } \\
\text { caso-control }\end{array}$ & Metaanálisis \\
\hline
\end{tabular}


Neurocirugía

Delgado-López y col

2005; 16: 142-157

\begin{tabular}{|c|c|c|c|c|}
\hline $\begin{array}{l}\text { Lahad A et } \\
\text { al, } 1994^{43}\end{array}$ & 64 estudios. & $\begin{array}{l}\text { En prevención de lumbalgia hay evidencia } \\
\text { limitada a favor de ejercicios de } \\
\text { fortalecimiento general y abdominal; } \\
\text { mínima en estrategias de educación; } \\
\text { insuficiente en uso de soportes mecánicos; } \\
\text { y nula en adelgazar o dejar de fumar }\end{array}$ & $\begin{array}{l}\text { Mayoría de } \\
\text { estudios en } \\
\text { ámbito laboral, } \\
\text { no clínico }\end{array}$ & Metaanálisis \\
\hline $\begin{array}{l}\text { Van Dieen JH } \\
\text { et al, } 1999^{68}\end{array}$ & ¿? & $\begin{array}{l}\text { La Squat Lifting Technique (cuclillas) frente } \\
\text { a Stoop (agacharse) produce menos } \\
\text { presión intradiscal pero no parece prevenir } \\
\text { lumbalgia; quizá sólo si se carga el peso } \\
\text { colocado entre los pies }\end{array}$ & $\begin{array}{l}\text { Sólo abstract } \\
\text { disponible }\end{array}$ & $\begin{array}{l}\text { Revisión } \\
\text { cualitativa }\end{array}$ \\
\hline $\begin{array}{l}\text { Staal JB et } \\
\text { al, } 2002^{61}\end{array}$ & $\begin{array}{l}14 \text { RCTs, } \\
\text { evalúan } 19 \\
\text { RTWs }\end{array}$ & $\begin{array}{l}\text { Las RTW (Return to Work interventions) } \\
\text { palian el coste del absentismo laboral por } \\
\text { lumbalgia. Son ejercicios físicos, } \\
\text { programas de educación y medidas de } \\
\text { ergonomía laboral }\end{array}$ & $\begin{array}{l}\text { Se describen las } \\
\text { medidas más } \\
\text { utilizadas pero no } \\
\text { su efectividad } \\
\text { clínica }\end{array}$ & Metaanálisis \\
\hline $\begin{array}{l}\text { Miltner O et } \\
\text { al, } 2001^{47}\end{array}$ & $\begin{array}{l}21 \text { estudios, } \\
1100 \text { pacientes. }\end{array}$ & $\begin{array}{l}\text { Eficacia indeterminada de ejercicios de } \\
\text { extensión lumbar en tratamiento de } \\
\text { lumbalgia }\end{array}$ & $\begin{array}{l}\text { Ningún estudio de } \\
\text { clase A. Artículo en } \\
\text { alemán. Sólo abst. }\end{array}$ & Metaanálisis. \\
\hline $\begin{array}{l}\text { Weinhardt C } \\
\text { et al, } 2001^{75}\end{array}$ & $\begin{array}{l}12 \mathrm{RCTs}, 9 \text { de } \\
\text { calidad }\end{array}$ & $\begin{array}{l}\text { El entrenamiento específico de músculos } \\
\text { de la espalda frente al entrenamiento físico } \\
\text { general obtiene resultados similares en } \\
\text { lumbalgia crónica y escaso beneficio frente } \\
\text { a no tratamiento o ejercicio pasivo }\end{array}$ & $\begin{array}{l}\text { Artículo en aleman. } \\
\text { Sólo abstract } \\
\text { disponible }\end{array}$ & Metaanálisis \\
\hline $\begin{array}{l}\text { Philaphelpia } \\
\text { Panel, 200154 }\end{array}$ & $\begin{array}{l}\text { Encuesta a } 324 \\
\text { médicos de } 6 \\
\text { organizaciones } \\
\text { profesionales. }\end{array}$ & $\begin{array}{l}\text { Se recomiendan como guías de práctica } \\
\text { clínica en lumbalgia: ejercicios lumbares } \\
\text { en dolor crónico, subagudo y post } \\
\text { quirúrgico; vuelta a actividad normal en } \\
\text { lumbalgia aguda) }\end{array}$ & $\begin{array}{l}\text { Sólo } 51 \% \text { de } \\
\text { respuestas }\end{array}$ & $\begin{array}{l}\text { Análisis de } \\
\text { datos de } \\
\text { encuesta }\end{array}$ \\
\hline $\begin{array}{l}\text { Schonstein E } \\
\text { et al, } 2003^{59}\end{array}$ & 18 RCTs. & $\begin{array}{l}\text { Ciertos programas de actividad física en el } \\
\text { medio laboral parecen reducir en } 45 \text { días el } \\
\text { tiempo de trabajo perdido por lumbalgia } \\
\text { crónica (no evidencia de efecto en } \\
\text { lumbalgia aguda) }\end{array}$ & $\begin{array}{l}\text { Seguimiento } \\
\text { de } 12 \text { meses. }\end{array}$ & $\begin{array}{l}\text { Revisión } \\
\text { Cochrane. } \\
\text { Metaanálisis }\end{array}$ \\
\hline $\begin{array}{l}\text { Flor H et al, } \\
1992^{19}\end{array}$ & 65 estudios. & $\begin{array}{l}\text { El tratamiento en centros de dolor } \\
\text { multidisciplinar es beneficioso y duradero } \\
\text { frente a no tratar o tratamiento } \\
\text { unidisciplinar en control del dolor lumbar, } \\
\text { vuelta al trabajo y consumo de recursos } \\
\text { médicos }\end{array}$ & $\begin{array}{l}\text { Mala calidad de } \\
\text { la mayoría de } \\
\text { los estudios }\end{array}$ & Metaanálisis \\
\hline $\begin{array}{l}\text { Fargas-Babjak } \\
\mathrm{A}, 2001^{17}\end{array}$ & $\begin{array}{l}6 \text { revisiones } \\
\text { sistemáticas y } \\
4 \text { RCTs }\end{array}$ & $\begin{array}{l}\text { Evidencia contradictoria o inadecuada } \\
\text { sobre la efectividad de la acupuntura, } \\
\text { TENS, laserterapia o neurorreflejoterapia } \\
\text { en lumbalgia crónica }\end{array}$ & $\begin{array}{l}\text { No compara contra } \\
\text { placebo y lo hace } \\
\text { contra acupuntura } \\
\text { sham }\end{array}$ & $\begin{array}{l}\text { Revisión de } \\
\text { estudios }\end{array}$ \\
\hline $\begin{array}{l}\text { Van der Weide } \\
\text { WE et al, } \\
1997^{67}\end{array}$ & 40 RCTs & $\begin{array}{l}\text { En lumbalgia aguda: evidencia limitada en } \\
\text { contra del reposo en cama y a favor de } \\
\text { breves períodos de reposo y manipulaciones } \\
\text { espinales. En lumbalgia crónica: evidencia } \\
\text { limitada a favor de los antidepresivos. }\end{array}$ & $\begin{array}{l}\text { Problemas de } \\
\text { aleatorización, } \\
\text { ciego y número } \\
\text { muestral en la } \\
\text { mayoría de los } \\
\text { estudios }\end{array}$ & Metaanálisis \\
\hline $\begin{array}{l}\text { Furlan AD et } \\
\text { al, } 2001^{24}\end{array}$ & 36 estudios & $\begin{array}{l}\text { Evidencia imprecisa o contradictoria sobre } \\
\text { la efectividad de } 19 \text { terapias conservadoras } \\
\text { en lumbalgia crónica. Sólo } 56 \% \text { de estudios } \\
\text { con conclusiones positivas. }\end{array}$ & $\begin{array}{l}\text { Los estudios de } \\
\text { resultado negativo } \\
\text { o impreciso eran } \\
\text { de mejor calidad }\end{array}$ & Metaanálisis \\
\hline
\end{tabular}




\begin{tabular}{|l|l|l|l|l|}
\hline $\begin{array}{l}\text { Van Tulder } \\
\text { MW et al, }\end{array}$ & $\begin{array}{l}\text { Estudios desde } \\
2000^{72}\end{array}$ & $\begin{array}{l}\text { Evidencia fuerte a favor de ejercicios físicos } \\
\text { y tratamiento multidisciplinar y en contra de } \\
\text { las tracciones en lumbalgia crónica. }\end{array}$ & $\begin{array}{l}\text { Estudio en } \\
\text { holandés. } \\
\text { Sólo se } \\
\text { Evidencia moderada a favor de AINEs, } \\
\text { escuela de la espalda y tratamiento } \\
\text { psicológico en lumbalgia crónica. }\end{array}$ & $\begin{array}{l}\text { Metaanálisis } \\
\text { abstract }\end{array}$ \\
& & & \\
\hline
\end{tabular}

(RCT: Ensayo clínico aleatorizado y controlado. VAS: Escala Analógico Visual del dolor. ACDF: Discectomía y fusión cervical anterior. OR: Odds Ratio).

Tabla 3

Tabla de Recomendaciones según MBE

\begin{tabular}{|c|c|c|}
\hline \multicolumn{3}{|c|}{ Nivel de evidencia } \\
\hline $\begin{array}{l}\text { La descompresión y extracción precoz del fragmento discal } \\
\text { mejora la recuperación sensitiva, motora y esfinteriana en } \\
\text { síndrome de cola de caballo por extrusión discal }{ }^{1}\end{array}$ & A & $\begin{array}{l}\text { No parece haber diferencia entre la cirugía antes de } 24 \text { horas } \\
\text { y entre } 24 \text { y } 48 \text { horas }\end{array}$ \\
\hline $\begin{array}{l}\text { La discectomía simple en ciática por prolapso discal lumbar } \\
\text { resistente a tratamiento conservador alivia el dolor en el } \\
70-95 \% \text { con efecto duradero hasta } 24 \text { meses }^{25,32}\end{array}$ & $\mathrm{~B}$ & $\begin{array}{l}\text { No hay evidencia de que altere de forma significativa la } \\
\text { historia natural de la enfermedad a largo plazo (evidencia D) }\end{array}$ \\
\hline $\begin{array}{l}\text { La quimionucleolisis con papaína es superior a placebo y su } \\
\text { efecto parece ser duradero hasta } 10 \text { años, pero inferior a } \\
\text { discectomía }{ }^{25}\end{array}$ & A & $\begin{array}{l}\text { Las complicaciones de la quimionucleolisis son menores a las } \\
\text { de la discectomía simple (evidencia C) }\end{array}$ \\
\hline $\begin{array}{l}\text { La vuelta al trabajo habitual tras discectomía ocurre en el } 80 \% \\
\text { (similar en el resto de procedimientos) }\end{array}$ & $\mathrm{C}$ & $\begin{array}{l}\text { Sólo } 59 \% \text { vuelven al trabajo tras múltiples cirugías } \\
\text { de la espalda }\end{array}$ \\
\hline $\begin{array}{l}\text { Microdiscetomía y discectomía simple obtienen resultados } \\
\text { similares o levemente favorables a la primera en prolapso y } \\
\text { espondilosis lumbar }{ }^{30,42,58,74}\end{array}$ & A & $\begin{array}{l}\text { Usar microscopio parece alargar levemente el tiempo } \\
\text { quirúrgico pero con igual morbilidad (evidencia C) y misma } \\
\text { formación de fibrosis (evidencia B) }\end{array}$ \\
\hline $\begin{array}{l}\text { Interponer membranas reduce levemente la formación de } \\
\text { cicatriz (fibrosis), lo que no parece influir positivamente en } \\
\text { el resultado-clínico }{ }^{44,53,74}\end{array}$ & $\mathrm{~B}$ & $\begin{array}{l}\text { Es dudoso que influya en la tasa de reoperaciones } \\
\text { (evidencia C) }\end{array}$ \\
\hline $\begin{array}{l}\text { La discectomía percutánea obtiene peores resultados clínicos } \\
\text { que la discectomía clásica }{ }^{1325,58}\end{array}$ & $\mathrm{C}$ & $\begin{array}{l}\text { No parecen influir las dosis de papaína, ésta comparada con } \\
\text { colagenasa, o ésta comparada con placebo (evidencia C). No } \\
\text { evidencia de efectividad de la discetomía láser (evidencia D) }\end{array}$ \\
\hline $\begin{array}{l}\text { Ausencia de efectividad probada de cualquier forma de fusión } \\
\text { o descompresión quirúrgica para espondilosis degenerativa } \\
\text { frente a placebo, tratamiento conservador o historia } \\
\text { natural }{ }^{25,27,46,64}\end{array}$ & A & Conclusión del metaanálisis de Gibson y cols (Cochrane) \\
\hline $\begin{array}{l}\text { En estenosis lumbar degenerativa de menos de } 8 \text { años de } \\
\text { evolución es preferible la descompresión sin fusión }\end{array}$ & $\mathrm{C}$ & $\begin{array}{l}\text { En más de } 15 \text { años de evolución se recomienda añadir fusión } \\
\text { instrumentada (evidencia C) }\end{array}$ \\
\hline $\begin{array}{l}\text { Ausencia de eficacia de la descompresión quirúrgica en } \\
\text { discopatía degenerativa o estenosis lumbar }{ }^{25}\end{array}$ & $\mathrm{D}$ & $\begin{array}{l}\text { No hay evidencia de que ninguna forma de cirugía en } \\
\text { discopatía degenerativa favorezca la vuelta al trabajo (evid D) }\end{array}$ \\
\hline $\begin{array}{l}\text { Las fusiones instrumentadas aumentan la tasa de fusiones } \\
\text { (aunque no hay consenso en cómo se mide la fusión } \\
\text { radiológica) } \\
\text { clínicos }^{815,49,74} \text { pero no se traduce en mejoría de los resultados }\end{array}$ & A & $\begin{array}{l}\text { La fusión vía anterior o posterior parecen iguales } \\
\text { (evidencia C) y la estimulación eléctrica favorece la fusión } \\
\text { (evidencia C) pero no mejora la clínica (evidencia B) }\end{array}$ \\
\hline $\begin{array}{l}\text { Descompresión y fusión es tan efectiva como fusión sola en } \\
\text { espondilolistesis ístmica grados I-II sin déficits }{ }^{14}\end{array}$ & $\mathrm{C}$ & \\
\hline $\begin{array}{l}\text { La descompresión sola es tan efectiva como descompresión } \\
\text { más fusión en listesis degenerativa }{ }^{74}\end{array}$ & $\mathrm{C}$ & \\
\hline $\begin{array}{l}\text { La cirugía de la radiculopatía cervical de un nivel por } \\
\text { espondilosis con o sin protrusión discal (blanda o dura) no es } \\
\text { mejor que el tratamiento conservador }{ }^{20,57,74}\end{array}$ & $\mathrm{~B}$ & $\begin{array}{l}\text { No está claro qué técnica es de elección: discectomía simple, } \\
\text { con fusión o con placa cervical (evidencia B) }\end{array}$ \\
\hline
\end{tabular}




\begin{tabular}{|c|c|c|}
\hline $\begin{array}{l}\text { En radiculopatía o mielopatía cervical espondilática, la cirugía } \\
\text { de descompresión es superior a la fisioterapia o al collarín } \\
\text { cervical, pero el efecto se iguala al año (radiculopatía) o a los } \\
\text { dos años (mielopatía con síntomas neurológicos leves) }{ }^{21}\end{array}$ & A & Conclusión del metaanálisis de Fouyas y cols (Cochrane) \\
\hline $\begin{array}{l}\text { Ausencia de efectividad de la cirugía en el dolor cervical } \\
\text { persistente por latígazo cervica } 1^{25,14}\end{array}$ & $\mathrm{D}$ & \\
\hline $\begin{array}{l}\text { La foraminoplastia endoscópica por láser presenta menos } \\
\text { complicaciones a corto plazo que las técnicas abiertas en } \\
\text { lumbalgia crónica y ciática }{ }^{37}\end{array}$ & $\mathrm{C}$ & \\
\hline $\begin{array}{l}\text { La antibioterapia profiláctica en cirugía de la columna reduce } \\
\text { a un tercio la tasa de infeccionesa }{ }^{6}\end{array}$ & $\mathrm{~B}$ & $\begin{array}{l}\text { Independientemente de la tasa basal de infección, tipo y dosis } \\
\text { de antibiótico }\end{array}$ \\
\hline $\begin{array}{l}\text { Eficacia del tratamiento fisioterápico en dolor cervical } \\
\text { mecánico del adulto }{ }^{28}\end{array}$ & $\mathrm{~B}$ & $\begin{array}{l}\text { Evidencia a favor de la estimulación eléctrica o } \\
\text { electromagnética y en contra de la laserterapia (evidencia C) }\end{array}$ \\
\hline $\begin{array}{l}\text { La estimulación epidural alivia al } 60 \% \text { de los pacientes con } \\
\text { Failed-Back o lumbalgia crónica } \\
65,66\end{array}$ & $\mathrm{~B}$ & $\begin{array}{l}\text { Presentan múltiples complicaciones menores relativas } \\
\text { a la técnica }\end{array}$ \\
\hline $\begin{array}{l}\text { La neurorreflejoterapia tiene efecto beneficioso sobre el dolor, } \\
\text { gasto en consultas y medicamentos al menos a corto } \\
\text { o medio plazo }{ }^{40,41}\end{array}$ & $\mathrm{~B}$ & $\begin{array}{l}\text { Técnica probada contra placebo en dolor de corta duración } \\
\text { (evidencia B) }\end{array}$ \\
\hline $\begin{array}{l}\text { La acupuntura es dudoso que produzca beneficio en lumbalgia } \\
\text { o cervicalgia crónica }{ }^{16,60,70}\end{array}$ & $\mathrm{~B}$ & $\begin{array}{l}\text { Parece superior a otros tratamientos controles pero } \\
\text { dudosamente superior a placebo (evidencia B) }\end{array}$ \\
\hline $\begin{array}{l}\text { Las manipulaciones espinales en lumbalgia aguda o crónica } \\
\text { son superiores sólo a placebo pero iguales a analgésicos, } \\
\text { ejercicios y escuela de la espaldas }\end{array}$ & $\mathrm{B}$ & \\
\hline El TENS no es superior a placebo ${ }^{10,74}$ & A & \\
\hline $\begin{array}{l}\text { Los antidepresivos en lumbalgia crónica disminuyen la } \\
\text { intensidad del dolor pero no mejoran la funcionalidad } \\
\text { del paciente }{ }^{56}\end{array}$ & A & \\
\hline $\begin{array}{l}\text { Las infiltraciones de corticoides epidurales en lumbalgia } \\
\text { crónica no parecen ser efectivas a medio plazo }\end{array}$ & A & \\
\hline $\begin{array}{l}\text { La denervación por radiofrecuencia no es efectiva en dolor } \\
\text { discogénico lumbar ni en cervicalgia }{ }^{51}\end{array}$ & $\mathrm{C}$ & Dudoso en síndrome facetario lumbar (evidencia C) \\
\hline $\begin{array}{l}\text { La posibilidad de obtener compensación económica por dolor } \\
\text { lumbar aumenta el dolor referido y disminuye la eficacia } \\
\text { del tratamiento }^{55}\end{array}$ & $\mathrm{C}$ & \\
\hline $\begin{array}{l}\text { La escuela de la espalda tiene una eficacia de corta } \\
\text { duración o nula en dolor crónico }\end{array}$ & $\mathrm{B}$ & $\begin{array}{l}\text { Quizá la variante sueca (más intensiva y sistemática) } \\
\text { sea beneficiosa }\end{array}$ \\
\hline $\begin{array}{l}\text { No existe asociación clara entre postura sentada en el trabajo } \\
\text { u ocupación sedentaria y lumbalgia crónica }{ }^{29,71}\end{array}$ & $\mathrm{~B}$ & \\
\hline $\begin{array}{l}\text { La exposición de todo el cuerpo a vibraciones intensas } \\
\text { predispone a la lumbalgia y a cambios degenerativos espinales }\end{array}$ & $\mathrm{C}$ & \\
\hline $\begin{array}{l}\text { Los soportes lumbares son eficaces en disminuir la movilidad } \\
\text { del tronco pero su efecto sobre el dolor no está demostrado }{ }^{69}\end{array}$ & $\mathrm{C}$ & \\
\hline $\begin{array}{l}\text { El fortalecimiento muscular general y de la espalda y la } \\
\text { educación de la espalda pueden prevenir la lumbalgia crónica }{ }^{43,72}\end{array}$ & $\mathrm{C}$ & $\begin{array}{l}\text { Nula evidencia a favor de adelgazar o dejar de fumar como } \\
\text { preventivos (evidencia D) }\end{array}$ \\
\hline $\begin{array}{l}\text { Las intervenciones educativas en el medio laboral reducen el } \\
\text { número de días perdidos de trabajo por dolor } \text { crónico }^{59,61}\end{array}$ & $\mathrm{~B}$ & \\
\hline $\begin{array}{l}\text { El tratamiento del dolor crónico en Centros de Dolor con } \\
\text { personal multidisciplinar parece beneficioso }{ }^{19,72}\end{array}$ & $\mathrm{~B}$ & $\begin{array}{l}\text { Afecta positivamente al número de bajas laborales y } \\
\text { consumo de recursos (evidencia B) }\end{array}$ \\
\hline
\end{tabular}


(evidencia moderada). No parecen existir diferencias marcadas entre los resultados de la discectomía clásica y la microdiscectomía, al menos en la cirugía del prolapso disca $1^{30,42,74}$ (evidencia fuerte), quizá a favor de la última ${ }^{56}$. Utilizar el microscopio podría alargar el tiempo quirúrgico ${ }^{42}$ (evidencia limitada) pero no parece influir en la formación de fibrosis, tiempo de hospitalización o pérdida de sangre $^{74}$ (evidencia moderada). La quimionucleolisis con papaína es superior al placebo, pero inferior a la discectomía clásica ${ }^{25,74}$ (evidencia fuerte). Los pacientes con síndrome de cola de caballo causado por extrusión discal se benefician de cirugía de descompresión precoz (antes de 48 horas) en términos de recuperación neurológica ${ }^{1}$ (evidencia fuerte). La interposición de membranas reduce levemente la formación de cicatriz (fibrosis peridural) pero no parece influir en el resultado clínico (evidencia moderada) y dudosamente afecta a la tasa de reoperaciones (evidencia limitada) ) $^{4,53,74}$.

Los pacientes con estenosis lumbar degenerativa se benefician de laminectomía descompresiva tanto como de laminectomía más fusión ${ }^{27,36}$ (evidencia fuerte), especialmente cuando ésta no es instrumentada ${ }^{15,36,74}$ (evidencia limitada). En estenosis lumbar con espondilolistesis degenerativa, la laminectomía más fusión parece ser superior a la laminectomía sola ${ }^{15,18,31}$ (evidencia fuerte). Los pacientes adultos con espondilolistesis ístmica se benefician de cirugía de fusión vertebral frente a tratamiento conservador ${ }^{48,74}$ (evidencia fuerte).

En dolor discogénico el tratamiento quirúrgico no parece superar al tratamiento conservador ${ }^{34}$ (evidencia fuerte) o en todo caso el beneficio es leve o moderado ${ }^{23}$.

En pacientes con hernia discal cervical y radiculopatía, la discectomía más fusión no parece superar a la

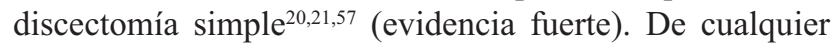
manera, el resultado quirúrgico no es mejor que la historia natural de la enfermedad o el tratamiento conservador más allá del primer o segundo año ${ }^{21}$.

La fusión vertebral radiológica podría obtenerse más frecuentemente con ayuda de instrumentación ${ }^{15}$ (evidencia moderada). Por otro lado, no parece existir una correlación evidente y demostrada entre fusión radiológica y alivio del dolor $^{8}$. La fusión vertebral se asocia a mayor tasa de complicaciones peroperatorias frente a la laminectomía simple, en términos de lesiones nerviosas, pérdida de sangre, necesidad de transfusión y mortalidad precoz ${ }^{8}$ (evidencia moderada). La tasa de reintervención es mayor en pacientes en los que se realiza una fusión instrumentada que en aquellos fusionados sin fijación y más en ésta que en los tratados mediante laminectomía simple ${ }^{45}$.

Los tornillos pediculares tienen un papel reconocido en el manejo de listesis, fracturas, luxaciones, tumores y pseudoartrosis ${ }^{8,15,72}$. Sin embargo, no parecen aportar un beneficio clínico evidente en la patología degenerativa frente a la fusión sola y aumentan la razón costebeneficio ${ }^{7,36}$. Sí parece que aumentan la tasa de fusiones radiológicas, así como la morbilidad postoperatoria ${ }^{7,62}$.

Los discos artificiales lumbares todavía no han sido suficientemente estudiados y su impacto sobre la EDE no ha demostrado un beneficio por encima de otros tratamientos $^{14}$. Los discos artificiales cervicales no han sido estudiados a nivel de metaanálisis hasta la fecha.

La utilización profiláctica de antibióticos en cirugía espinal parece reducir a un tercio la tasa de infecciones postoperatorias independientemente de la tasa de incidencia de base y de si se extiende la cobertura a gérmenes gram negativos además de los gram positivos, o de si se emplean dosis únicas o múltiples de antibióticos ${ }^{6}$.

\section{Tratamientos no quirúrgicos}

Multitud de procedimientos y medicamentos se han empleado en el dolor de la EDE. Ninguna de estas medidas está avalada por estudios que permitan emitir recomendaciones basadas en evidencia fuerte ${ }^{71}$. La mayoría de los beneficios que se obtienen de estas terapias suelen ser parciales y de duración limitada.

Se descarta la utilidad del reposo en cama tanto en dolor agudo como crónico ${ }^{67,71}$. Los ejercicios de fortalecimiento lumbar ${ }^{47,59,72,75}$ y los programas de educación e higiene postural $1^{59,61,68}$ en el ámbito laboral o en las "escuelas de la espalda" parecen ser de algún modo beneficiosos siempre que se realicen en determinadas condiciones y de forma intensiva ${ }^{39,72}$. Aun así, no parecen prevenir la lumbalgia ${ }^{43}$. La postura forzada ${ }^{29}$, la exposición a vibraciones intensas ${ }^{9}$ y el sedentarismo ${ }^{29}$, no están vinculados de forma evidente a una peor evolución del dolor.

Otros procedimientos muchas veces testados pero con eficacia dudosa o limitada en el tiempo son el TENS ${ }^{10,17}$, la acupuntura ${ }^{16,60,70}$, la estimulación epidural ${ }^{65,66}$, las manipulaciones espinales ${ }^{5}$, las infiltraciones epidurales ${ }^{38}$ y los refuerzos lumbares ${ }^{69}$. Es dudoso o nulo el efecto de dejar de fumar o adelgazar ${ }^{43}$. Los antidepresivos disminuyen la percepción del dolor pero no mejoran la funcionalidad del paciente ${ }^{56}$ (evidencia fuerte). La neurorreflejoterapia parece aportar beneficio cuando se añade al tratamiento convencional, al menos a corto plazo ${ }^{40,41}$ (evidencia moderada). Técnicas como la denervación por radiofrecuencia ${ }^{51}$ o la foraminoplastia endoscópica por láser ${ }^{37}$ no obtienen resultados concluyentes ni contrastados. No existen revisiones sistematizadas sobre ozonoterapia intradiscal de las que obtener conclusiones.

Es interesante mencionar la relación inversa entre respuesta al tratamiento del dolor crónico y la posibilidad de obtener compensación económica ${ }^{55}$. Este efecto ya se ha evidenciado en patologías controvertidas como el latigazo cervical $^{50}$. 


\section{Discusión}

La medicina basada en la evidencia (MBE) es, actualmente, la herramienta metodológica más empleada a la hora de establecer relaciones causales en Ciencias de la Salud y es el marco de trabajo que permite la toma de decisiones sobre una base científica. El metaanálisis, o revisión sistematizada de estudios, proporciona una magnitud del efecto de una determinada intervención tanto cualitativa como cuantitativa, con el máximo control de sesgos. Combina los resultados de diversos estudios y clasifica las intervenciones en función de la fuerza de evidencia científica que se extrae del análisis. Los niveles de evidencia clasifican las recomendaciones en estándares de tratamiento, guías de práctica clínica o simplemente opciones terapéuticas. En lo referente a la EDE, lamentablemente disponemos de muy pocas recomendaciones firmes a nivel de estándares de tratamiento debido a la defectuosa calidad de los estudios. En principio, la efectividad de un tratamiento debe compararse contra la ausencia de tratamiento (historia natural de la enfermedad) o contra placebo bien enmascarado.

\section{Relevancia de la enfermedad degenerativa espinal}

Nachemson y cols publicaron en 2000 una extensa revisión sobre el tema ${ }^{49}$, aplicando criterios de MBE, y haciendo hincapié en aspectos como la etiopatogenia, evaluación, manejo y prevención de la EDE y el dolor de espalda y cuello en general, así como en sus implicaciones sociales y costes. Los datos socio-epidemiológicos referidos en los párrafos a continuación se han extractado de dicha monografía, que recomendamos por su claridad y meticulosidad $^{49,50}$.

Desde el punto de vista demográfico y epidemiológico, el dolor de espalda crónico afecta por igual a ambos sexos (quizá algo más prevalente en el hombre a nivel global), a cualquier edad incluida la pediátrica (con inicio sobre todo entre la $3^{\text {a }}$ y $5^{\text {a }}$ décadas), afecta a todas las razas y estratos socioculturales $\mathrm{y}$, clásicamente, se ha asociado a trabajos, posturas y ocupaciones donde la unidad musculoligamentosa axial de la columna está de alguna manera comprometida o forzada.

La prevalencia puntual del dolor lumbar se estima en torno al $15-30 \%$ de toda la población. Alrededor del 19$43 \%$ de las personas de un país lo han sufrido en mayor o menor intensidad en el último mes. Entre un $60-80 \%$ de las personas sufrirán dolor lumbar incapacitante alguna vez en su vida. El dolor de espalda es la primera causa de "enfermedad crónica" en hombres y mujeres menores de 64 años y la segunda entre 65 y 74 años, tras los problemas circulatorios. Los problemas de la espalda son la primera causa de limitación en la actividad laboral en menores de 45 años y la cuarta entre los de edades comprendidas entre 45 y 64 años.

Entre el 7-14\% de los norteamericanos pierden un día de trabajo cada año por dolor de espalda y el $1 \%$ están impedidos de forma continua (otro $1 \%$ de forma temporal). En 1988 se perdieron en EE.UU. 149 millones de días de trabajo como consecuencia del dolor lumbar que padecieron 22,4 millones de norteamericanos (el 17,6\% de todos los trabajadores). En 1993 se perdieron en el Reino Unido 52 millones de días de trabajo y se pagaron 106 millones de días de baja e invalidez por esta causa. La mitad de los días perdidos por dolor de espalda tienen como protagonistas al $85 \%$ de las personas que se toman bajas laborales de corta duración (menos de 7 días). La otra mitad corresponden al $15 \%$ de trabajadores que permanecen en casa por lo menos un mes. El $80-90 \%$ de los costes médicos y sociales del dolor lumbar están relacionados con el $10 \%$ de los pacientes más crónicos.

La prevalencia a lo largo de la vida del dolor ciático (entendido como dolor referido a la pierna y no sólo la radiculopatía evidente) se estima en torno al $14-40 \%$ de la población. Con criterios más estrictos, la ciática afecta en torno al $4-5 \%$ de la población a lo largo de la vida. La prevalencia a lo largo de la vida de la hernia discal de interés neuroquirúrgico se estima en torno al $2 \%$ únicamente (quizá algo mayor en hombres y algo menor en mujeres).

En cuanto a la patología cervical, la prevalencia puntual del dolor cervical se estima entre el $11-22 \%$ de la población; entre el $10-40 \%$ en los últimos 6 meses; entre el $18-40 \%$ al año, y a lo largo de la vida en torno al $67-71 \%$. Parece ser mayor en ciertas profesiones como dentistas, mineros o secretarias donde se aducen razones posturales.

De todas las lesiones relacionadas con el trabajo en EE.UU., un tercio tienen que ver con problemas de la espalda (se presentan en torno a un millón de reclamaciones de compensación económica anualmente en EE.UU.). No parece haberse documentado un aumento en la incidencia o prevalencia de dolor lumbar a lo largo de la historia reciente (últimos 20 años), aunque sí ha crecido el número de declaraciones de invalidez por dolor lumbar o cervical inespecíficos.

\section{Controversias acerca de la cirugía de fusión espinal}

La cirugía de la columna vertebral plantea siempre tres cuestiones que el cirujano debe tener en mente cuando se indica una intervención, como son: a) la necesidad y grado de descompresión de las estructuras nerviosas (médula y/o raíces nerviosas); b) la necesidad y tipo de abordaje para realizar una fusión (artrodesis) vertebral; y c) la recomendación o no de reforzar la artrodesis con una instrumentación (fijación interna). La Tabla de Recomendaciones según MBE reúne las recomendaciones terapéuticas según su nivel de evidencia y pueden ser de interés a la hora de 
tomar decisiones en estos tres términos comentados.

En tiempos recientes se ha constatado una llamativa tendencia hacia la realización de procedimientos de fusión vertebral para procesos diferentes a los que inicialmente constituían su indicación, esto es, fracturas vertebrales, tumores, luxaciones, pseudoartrosis, listesis y otras deformidades ${ }^{8}$. Actualmente, el $75 \%$ de las fusiones espinales se indican en pacientes con espondilosis, discopatías y estenosis de canal ${ }^{8,15}$. En EE.UU., mientras la cirugía de prótesis de cadera o la artroplastia de rodilla ha aumentado un 13$14 \%$ entre 1996 y 2001, la cirugía de fusión espinal se ha incrementado un $77 \%$ en el mismo período. Este mercado de implantes espinales mueve en torno a 2.000 millones de dólares cada año, con un incremento medio anual del $18-20 \%$ (el coste medio por procedimiento en ese país es de 34.000 dólares, excluyendo los honorarios profesionales $)^{15}$.

Entre la década de los ochenta y los noventa se ha doblado el número de fijaciones internas espinales ${ }^{8,15,50}$. Las razones de este aumento pueden estar en un conjunto de factores como son el envejecimiento poblacional, la aparición de nuevos injertos óseos (materiales osteoinductores y osteoconductores) y cajas intersomáticas (aprobados por la FDA norteamericana desde 1996), técnicas quirúrgicas más depuradas y menos invasivas (endoscópicas y mínimamente invasivas) y, quizá, incentivos comerciales que interesan a centros hospitalarios, personal facultativo y casas comerciales. Sin embargo, es de destacar que, si bien las cirugías de fusión vertebral se instrumentaron globalmente durante los años ochenta en el 23\% de las ocasiones y en los noventa en el $41 \%$ de los casos, este hecho no se ha traducido en una mejoría de los resultados clínicos, ni siquiera en un aumento de las tasas globales de fusión ${ }^{8}$.

A la vista de la lectura crítica de los metaanálisis arriba expuestos, la única recomendación claramente establecida de realizar fusión vertebral en la EDE parece ser la espondilolistesis ístmica del adulto que no responde al tratamiento conservador y la estenosis lumbar con espondilolistesis degenerativa de los últimos espacios discales. Paradójicamente, la mayor parte de las indicaciones de fusión vertebral no corresponden a este capítulo sino a discopatías y espondilosis diversas extendiéndose la indicación a procesos como el dolor discogénico.

Algo similar ha ocurrido con anterioridad en relación a la instrumentación transpedicular, que fue introducida en los años ochenta sin el aval de ensayos aleatorizados y controlados, ni siquiera con estudios de cohortes prospectivas ${ }^{15}$ (los tornillos pediculares no están autorizados en EE.UU. para la EDE). Sus indicaciones han variado a lo largo de los años de forma que actualmente acompañan a la mayoría de las fusiones por vía posterior, aun a sabiendas de que su utilización en la EDE no está validada por ningún estudio de clase A y aumentan positivamente la morbilidad y la tasa de reintervenciones ${ }^{7,15,45,62}$. En realidad, no parece coherente establecer la necesidad de fijar una columna degenerada basándose en la premisa de que, eventualmente se evitará tener que volver a intervenir al paciente por inestabilidad o dolor postlaminectomía, cuando la propia instrumentación se asocia a una mayor incidencia de reoperaciones.

Se han descrito diversas técnicas quirúrgicas de fusión vertebral lumbar en la EDE, procedimientos tales como las artrodesis intertransversas, laminares, espinosas, intersomáticas anteriores (ALIF), intersomáticas posteriores (PLIF) y transforaminales (TLIF) con diversos materiales autólogos y heterólogos. Muchos autores comunican resultados muy favorables y tasas bajas de morbilidad a medida que se gana experiencia con una técnica determinada. En nuestra opinión, no creemos tan relevante qué técnica de fusión se emplea como en qué paciente se realiza. La disponibilidad de una determinada técnica e incluso la maestría al realizarla no creemos que deban ser los factores que determinan su indicación. Sí debe serlo la demostración clínico-epidemiológica de que es efectiva para cada paciente con una patología concreta. Muchas veces no se dispone de tal evidencia, por lo que lo adecuado es participar en ensayos clínicos dirigidos a demostrar su efectividad. Desde el punto de vista ético, algunos autores empiezan a cuestionar la necesidad de realizar ensayos clínicos aleatorizados y ciegos de tratamientos quirúrgicos frente a "placebos quirúrgicos" ${ }^{133}$ (sham-surgery), pues se trata de una enfermedad que no supone, en principio, un riesgo para la vida, la tasa de complicaciones de las intervenciones puede ser alta, es una patología extremadamente relevante desde el punto de vista de la financiación sanitaria, y los resultados son difíciles de medir y ciertamente subjetivos ${ }^{15}$.

La magnitud socioeconómica de la EDE, ya expuesta, sugiere que las implicaciones financieras derivadas de los tratamientos son enormes ${ }^{15,50}$. Waddell resume y comenta de forma inteligente las controversias en torno a la instrumentación espinal ${ }^{73}$ : la instrumentación parece aumentar la tasa de fusión vertebral pero sólo de forma moderada; no existe un consenso claro sobre la forma de demostrar la fusión radiológica; fusión radiológica no implica necesariamente mejoría clínica; no se conoce a ciencia cierta qué subgrupo de pacientes sí mejoran de forma clara tras la fusión instrumentada; y la morbilidad y costes de la instrumentación, a día de hoy, no se comparan positivamente frente a la cirugía no instrumentada. Este autor advierte que, tarde o temprano, pacientes y terceras partes implicadas en la financiación sanitaria (¿o en el litigio?) podrían emplear estos argumentos para desacreditar la cirugía de fusión espinal en la mayoría de sus indicaciones; cuestión para la que no tenemos una contestación basada en evidencias científicas sólidas.

El análisis del seguimiento clínico y de las variables de resultado como son las escalas de medida del dolor y las 
escalas de funcionalidad no es objeto de este trabajo pero tampoco parece resuelto. La mayor parte de los pacientes intervenidos que no presentan complicaciones tras la cirugía son probablemente dados de alta tras una o dos revisiones a corto plazo por lo que la historia natural del paciente intervenido se conoce basándose en estudios transversales de corte (fundamentalmente encuestas telefónicas), con los sesgos que ello conlleva.

De forma análoga, los tratamientos no quirúrgicos de la EDE son de múltiples tipos y se emplean en la práctica diaria bajo evidencia escasa o nula en la mayor parte de los $\operatorname{casos}^{24}$. La Tabla de Recomendaciones según MBE (Tabla 3) pone de manifiesto el escaso apoyo científico que avala dichas terapias. El reposo absoluto en cama parece desterrado definitivamente de las recomendaciones en EDE, así como la utilización intensiva y prolongada de analgésicos, antiinflamatorios y relajantes musculares. Las medidas de higiene postural y de realización de esfuerzos físicos, y el fortalecimiento muscular general y de la columna no está demostrado que prevengan la lumbalgia pero se recomiendan en el tratamiento a largo plazo del paciente que sufre dolor de espalda. Así mismo, se recomienda retomar la actividad física y laboral lo antes posible tras la crisis de dolor, hasta donde éste lo permita. El TENS, acupuntura, quiropráctica, neurorreflejoterapia, estimulación epidural, fajas y corsés, infiltraciones epidurales y facetarias, y multitud de programas de educación en la biomecánica y prevención de las lesiones de la espalda no parecen ser efectivas por sí solas sino que, probablemente, se deban emplear en combinación, teniendo en cuenta que el alivio del dolor suele ser moderado y casi siempre transitorio. No disponemos de estudios de seguimiento a largo plazo de tratamientos como la ozonoterapia, actualmente en uso creciente para patología discal degenerativa lumbar y cervical.

\section{Conclusiones}

La cirugía de la EDE se asienta sobre pilares inseguros habida cuenta de que la mayor parte de las técnicas que se indican no están avaladas por recomendaciones de primera clase en términos de MBE. El llamativo incremento de las intervenciones de fusión espinal en procesos diferentes a las deformidades espinales y espondilolistesis aisladas o acompañadas de estenosis del canal lumbar, quizá obedece a múltiples factores técnicos y clínicoepidemiológicos donde no podemos obviar la enorme trascendencia económica que subyace.

Quizá estemos en disposición de consensuar guías de práctica clínica en lo referente al tratamiento integral y multidisciplinario de la EDE, a sabiendas que, hasta hoy, pocos tratamientos alteran de forma positiva y duradera la historia natural de la enfermedad. El conocimiento de la evolución de los pacientes con y sin tratamiento es crucial para entender el efecto relativo de las intervenciones que realizamos. La MBE es el instrumento básico con el cual debemos comparar la efectividad de los tratamientos.

Esta revisión y otras con anterioridad ${ }^{8,15,21,25,74}$ ponen de manifiesto una discrepancia entre práctica diaria y evidencia científica, por lo que creemos del máximo interés establecer, desde las organizaciones científicas pertinentes que estudian la columna degenerativa, las pautas de investigación clínica necesarias que controlen y aseguren la adecuación entre lo que se desprende del conocimiento clínico y la aplicación práctica de los diversos tratamientos.

\section{Bibliografía}

1. Ahn, U.M., Ahn, U.N., Buchowsky, J.M., Garrett, E.S., Sieber, AN., Kostuik, J.P.: Cauda equina symdrome secondary to lumbar disc herniation: a meta-analysis of surgical outcomes. Spine 2000; 25: 1515-1522.

2. Ahn, U.N., Ahn, U.M., Nallamshetty, L., et al.: Cauda equina syndrome in ankylosing spondylitis (the CES-AS syndrome): meta-analysis of outcomes after medical and surgical treatments. J Spinal Disord 2001; 14: 427-433.

3. Akai, M., Kawashima, M., Kimura, T., Hayashi, K.: Electrical stimulation as an adjunct to spinal fusion: a metaanalysis of controlled clinical trials. Bioelectromagnetics 2002; 232: 493-504 (abstract).

4. Assendelft, W.J., Koes, B.W., van der Heijden, G.J., Bouter, L.M.: The effectiveness of chiropractic for treatment of low back pain: an update and attempted at statistical pooling. J Manipulative Physiol Ther 1996; 19: 499-507.

5. Assendelft, W.J., Morton, S.C., Yu, E.I., Suttorp, M.J., Shekelle, P.G.: Spinal manipulative therapy for low back pain. A meta-analysis of effectiveness relative to other terapies. Ann Intern Med 2003; 138: 871-881.

6 . Barker, F., $2^{\text {nd }} .:$ Efficacy of prophylactic antibiotic therapy in spinal surgery: a meta-analysis. Neurosurgery 2002; 51: 391-400.

7. Bjarke Christensen, F., Stender Hansen, E., Laursen, M., Thomsen, K., Bunger,C.E.: Long-term functional outcome of pedicle screw instrumentation as a support for posterolateral spinal fusion: randomized clinical study with a 5-year followup. Spine 2002 ;27: 1269-1277.

8. Bono, C.M., Lee, C.K.: Critical analysis of trends in fusion for degenerative disc disease over the past 20 years: influence of technique on fusion rate and clinical outcome. Spine 2004; 29:455-463.

9. Bovenzi, M., Hulshof, C.T.: An update review of epidemiologic studies on the relationship between exposure to whole-body vibration and low back pain (1986-1997). Int Arch Occup Environ Health 1999;72:351-365 (abstract).

10. Brosseau, L., Milne, S., Robinson, V., et al.: Efficacy of transcutaneous electrical nerve stimulation for the treatment of chronic low back pain: a meta-analysis. Spine 2002; 27: 596-603. 
11. Burkus, J.K., Heim, S.E., Gornet, M.F., Zdeblick, T.A.: Is INFUSE bone graft superior to autograft bone? An integrated analysis of clinical trials usin the LT-CAGE lumbar tapered fusion device. J Spinal Disord Tech 2003; 16: 113-122 (abstract).

12. Caragee, E.J.: Single level posterolateral arthrodesis with or without posterior decompression for the treatment of isthmic spondylolisthesis in adults: a prospective, randomised study. J Sone Joint Surg Am 1997; 1175-1180.

13. Chatterjee, S., Foy, P.M., Findlay, G.F.: Report of a controlled clinical trial comparing automated percutaneous lumbar discectomy and microdiscectomy in the treatment of contained lumbar disc herniation. Spine 1995; 20:734-738.

14. De Kleuver, M., Oner, F.C., Jacobs, W.C.: Total disc replacement for chronic low back pain: background and a systematic review of the literature. Eur Spine J 2003; 12: 108116.

15. Deyo, R.A., Nachemson, A., Mirza, S. K.: Spinalfusion surgery - The case for restraint. N Engl J Med 2004; 350: $722-726$.

16. Ernst, E., White, A.R.: Acupuncture for low back pain: a meta-analysis of randomizaed controlled trials. Arch Intern Med 1998; 158: 2235-2241.

17. Fargas-Babjak, A.: Acupuncture, transcutaneous electrical nerve stimulation and laser therapy in chronic pain. Clin J Pain 2001; 17 (suppl): S105-113.

18. Fischgrund, J.S., Mackay, M., Herkowitz, H.M., Brower, R., Montgomery, D.M., Kurz, L.T.: Degenerative lumbar spondylolisthesis with spinal stenosis: a prospective randomized study comparing decompressive laminectomy and arthrodesis with and without instrumentation. Spine 1997; 22: 2807-2812.

19. Flor, H., Fydrich, T., Turk, D.C.: Efficacy of multidisciplinary pain treatment centers: a meta-analytic review. Pain 1992; 49: 221-230.

20. Floyd, T., Ohnmeiss D.: A meta-analysis of autograft versus allograft in anterior cervical fusion. Eur Spine J 2000. 9: 398-403.

21. Fouyas, I.P., Statham, P. F., Sandercock, P.A.: Cochrane review on the role of surgery in cervical spondylotic radiculomyelopathy. Spine 2002; 27: 736-747.

22. Fraser, R.D., Sandhu, A., Gogan, W.J.: The Magnetic Resonance imaging findings 10 years after treatment for lumbar disc herniation. Spine 1995; 20: 710-714.

23. Fritzell, P., Hagg, O., Wesberg, P., Nordwall, A.: Chronic low back pain and fusion: a comparison of three surgical techniques: a prospective multicenter randomized study from the Swedish Lumbar Spine Study Group. Spine 2002; 27: 1131-1141.

24. Furlan, A.D., Clarke, J., Esmail, R., Sinclair, S., Irvin, E., Bombardier, C.: A critical review of reviews on the treatment of chronic low back pain. Spine 2001; 26: E155-E162.

25. Gibson, J.N., Grant, LC, Waddell, G.: The Cochrane
Review of surgery for lumbar disc prolapse and degenerative lumbar spondylosis. Spine 1999; 24: 1820-1832.

26. Goldberg, M.S., Scott, S.C., Mayo, N.E.: A review of the association between cigarrette smoking and the development of non specific back pain and related outcomes. Spine 2000; 25: 995-1014.

27. Grob, D., Hunke, T., Dvorak, J.: Degenerative Lumbar spinal stenosis: decompression with and without arthrodesis. J Bone Surg Am 1995; 77: 1036-1041.

28. Gross, A.R., Aker, P.D., Goldsmith, C.H., Peloso, P.: Conservative management of mechanical neck disorders. A systematic overview and meta-analysis. Online J Curr Clin Trials 1996; Jul 30: Doc No 200-201.

29. Hartvigsen, J., Leboeuf-Yde, C., Lings, S., Corder, E.H.: Does sitting at work cause low back pain? Ugeskr Laeger 2002; 164: 759-761 (abstract).

30. Henrikson, L., Schmidt, V., Eskesen, V., et al.: A Controlled Study of microsurgical versus standard lumbar discectomy. Br J Neurosurg 1996; 10: 289-293.

31. Herkowitz, H. N., Kurz, L.T.: Degenerative lumbar spondylolisthesis with spinal stenosis: a prospective study comparing decompression with decompression and intertransverse process arthrodesis. J Bone Joint Surg Am 1991; 73: 802-808.

32. Hoffman, R.M., Wheeler, K.J., Deyo, R.A.: Surgery for herniated lumbar discs: a literature synthesis. J Gen Intern Med. 1993; 8: 487-496.

33. Horng, S., Miller, F.G.: Is placebo surgery unethical? N Engl J Med 2002; 347: 137-139.

34. Ivar Brox, J., Sorenson, R., Friis, A., et al.: Randomized clinical trial of lumbar instrumented fusion and cognitive intervention and exercises in patients with chronic low back pain and disc degeneration. Spine 2003; 28: 1913-1921.

35. Kant, A.P., Daum, W.J, Dean, S.M., et al.: Evaluation of lumbar spine fusion: plain radiographs versus direct surgical exploration and observation. Spine 1995; 20: 2313-2317.

36. Katz, J.N., Lipson, S.J., Lew, R.A.: Lumbar laminectomy alone or with instrumented or noninstrumented arthrodesis in degenerative lumbar spinal stenosis: patient selection, costs, and surgical outcomes. Spine 1997; 22: 1123-1131.

37. Knight, M.T., Ellison, D. R., Goswami, A., Hillier, V. F.: Review of safety in endoscopic laser foraminoplasty for the management of back pain. J Clin Laser Med Surg 2001; 19: 147-157 (abstract).

38. Koes, B.W., Scholten, R.J., Mens, J.M., Bouter, L.M.: Efficacy of epidural steroid injections for low back pain and sciatica: a systematic review of randomized clinical trials. Pain 1995; 63: 279-288.

39. Koes, B.W., van Tulder, M.W., van der Windt, W.M., Bouter, L.M.: The efficacy of back schools: a review of randomized clinical trials. J Clin Epidemiol 1994; 47: 851-862.

40. Kovacs, F. M., Abraira, V., Pozo F., et al.: Local and remote sustained trigger point therapy for exacerbations of 
chronic low back pain. A randomized, double blind, controlled multicenter trial. Spine 1997; 22: 786-797.

41. Kovacs, F.M., Llobera, J., Abraira, V., Lazaro, P., Pozo, F., Kleinbaum, D.: Effectiveness and cost-effectiveness analysis of neuroreflexotherapy for subacute and chronic low back pain in routine general practice: a cluster, randomized, controlled trial. Spine 2002; 27: 1149-1159.

42. Lagarriage, J., Chaines, P.: Comparative study of disc surgery with or without microscsopy: a prospective study of 80 cases. Neurochirurgie 1994; 40: 116-120.

43. Lahad, A., Malter, A.D., Berg, A.O., Deyo, R.A.: The effectiveness of four interventions for the prevention of low back pain. JAMA 1994; 272: 1286-1291.

44. MacKay, M.A., Fischgrund, J.S., Herkowitz, H.M., et al.: The effect of interposition membrane on the outcome of lumbar laminectomy and discectomy. Spine 1995; 20: 17931796.

45. Malter, A.D., McNeney, B., Loeser, J.D., Deyo, R.A.: 5 -year reoperation rates after different types of lumbar spine surgery. Spine 1998; 23: 814-820.

46. Mardjetko, S.M., Connolly, P.J., Shott, S.: Degenerative lumbar spondylosis: a meta-analysis of literature 19701993. Spine 1994; 20S: 2256S-2265S.

47. Miltner, O., Wirtz, D.C., Siebert, C.H.: Strengthening lumbar extensors therapy of chronic back pain: an overview and meta-analysis. Z Orthop Ihre Grenzgeb 2001; 139: $287-$ 293 (abstract).

48. Moller, H., Hedlund, R.: Surgery versus conservative management in adult isthmic spondylolisthesis - a prospective randomized study: part 1 . Spine 2000; 25: 1711-1715.

49. Nachemson, A., Jonsson, E.: Neck and back pain. The scientific evidence of causes, diagnosis and treatment. Philadelphia, Lippincott Williams \& Wilkins, 2000.

50. Nachemson, A., Waddell, G., Norlund, A.I.: Epidemiology of Neck and Back Pain. En Nachemson A (ed). Neck and back pain. The scientific evidence of causes, diagnosis and treatment. Philadelphia, Lippincott Williams \& Wilkins, 2000; pp.165-188.

51. Niemisto, L., Kalso, E., Malmivaara, A, Seitsalo, S., Hurri, H.: Radiofrequency denervation for neck and back pain. A systematic review of randomized controlled trials. Cochrane Database Syst Rev 2003;(1):CD004058.

52. Niggemeyer, O., Strauss, J.M., Schulitz, K.P.: Comparison of surgical procedures for degenerative lumbar spinal stenosis: a meta-analysis of the literature from 1975 to 1995. Eur Spine J 1997; 6: 423-429.

53. Petrie, J.L., Ross, J.S.: Use of ADCON-L to inhibit postoperative peridural fibrosis and related symptoms following lumbar dise surgery: a preliminary report. Eur Spine J 1996; 5 (suppl 1): S10-S17.

54. Philadelphia Panel.: Philadelphia Panel evidence-based clinical practice guidelines on selected rehabilitation interventions forlow back pain. Phys Ther 2001; 81: 641-1674.
55. Rohling, M. L., Binder,L.M., LanghinrischsenRohling, J.: Money matters: a meta-analytic review of the association between financial compensation and the experience and treatment of chronic pain. Health Psychol 1995; 14: 537-547.

56. Salerno, S.M., Browning, R., Jackson, J.L.: The effect of antidepressant treatment on chronic back pain: a metaanalysis. Arch Intern Med 2002; 162: 19-24.

57. Savolainen, S., Rinne, J., Hernesniemi, J.: A prospective randomized study of anterior single-level cervical disc operations with long-term follow-up: surgical fusion is unnecessary. Neurosurgery 1998; 43: 51-55.

58. Schmid, U.D.: Microsurgery of lumbar dise prolapse. Superior results of microsurgery as compared to standard and percutaneous procedures (review of literature). Nervenartz 2000; 71: 265-274 (abstract).

59. Schonstein, E., Kenny, D.T., Keating, J., Koes, B.W.: Work conditioning, work hardening and functional restoration for workers with back and neck pain. Cochrane Database Syst Rev 2003; (1): CD001822.

60. Smith, L.A., Oldman, A. D., McQuay, H.J., Moore, R.A.: Teaasing apart quality and validity in systematic reviews: an example from acupuncture trials in chronic neck and back pain. Pain 2000; 86: 119-132.

61. Staal, J.B., Hlobil, H., van Tulder, M.W., Koke, A.J., Smid, T., van Mechelen, W.: Return-to-work interventions for low back pain: a descriptive review of contents and concepts of working mechanisms. Sports Med 2002; 32: 251-267.

62. Thomsen, K., Christensen, F.B., Eiskjaer, S.P., Hansen, E.S., Fruensgaard, S., Bunger, C.E.; The effect of pedicle screw instrumentation on functional outcome and fusion rates in posterolateral lumbar spinal fusion: a prospective randomized clinical study. Spine 1997; 22: 2813-2822.

63. Turner, J.A., Ersek, M., Herron, L., Deyo, R.: Surgery for lumbar spinal stenosis. Attempted meta-analysis of the literature. Spine 1992; 17: 1-8.

64. Turner, J.A., Herron, L., Deyo, R.A.: Meta-analysis of results of lumbar spine fusion. Acta Orthop Scand 1993; 64 (suppl 251): 120-122.

65. Turner, J.A., Loeser, J.D., Bell, K.G.: Spinal cord stimulation for chronic low back pain: a systhematic literature synthesis. Neurosurgery 1995; 37: 1088-1095.

66. Van Buyten, J.P., Van Zundert, J., Vueghs, P., Vanduffel, L.: Efficacy of spinal cord stimulation: 10 years of experience in a pain center in Belgium. Eur J Pain 2001; 5: 299-307.

67. Van der Weide, W. E., Verbeek, J.H., van Tulder, M.W.: Vocational outcome of intervention for low back pain. Scand J Work Environ Health 1997; 23: 165-178 (abstract).

68. Van Dieen, J.H., Hoozemans, M.J., Toussaint, H.M.: Stoop and squat: a review of biomechanical studies on lifting technique. Clin Biomech 1999; 14: 685-696 (abstract).

69. Van Poppel, M. N., de Looze, M. P., Koes, B.W., Smid, 
T., Bouter, L.M.: Mechanisms of action of lumbar supports: a systematic review. Spine 2000; 25: 2103-2113.

70. Van Tulder, M.W., Cherkin, D.C., Berman, B., Lao, L., Koes, B.W.: The effectiveness of acupuncture in the management of acute and chronic low back pain. A systematic review within the framework of the Cochrane Collaboration Back Review Group. Spine 1999; 24: 1113-1123.

71. Van Tulder, M.W., Goosens, M., Waddell, G., Nachemson, A.: Conservative treatment of chronic lowback pain. En Nachemson A (ed). Neck and back pain. The scientific evidence of causes, diagnosis and treatment. Philadelphia, Lippincott Williams \& Wilkins, 2000; pp.271-304.

72. Van Tulder, M.W., Koes, B.W., Assendelft, W.J., Bouter, L.M., Maljers, L.D., Driessen, A.P.: Chronic low back pain: exercise therapy, multidisciplinary programs, NSAID's, back schools and behavioral therapy effective; traction not effective; results od systematic reviews. Ned Tijdschr Geneeskd 2000; 144: 1489-1494 (abstract).

73. Waddell, G.: Point of view. Spine 2004; 2925 (letter). 74. Waddell, G., Gibson, J.N.A., Grant, I.: Surgical Treat- ment of lumbar disc prolapse and degenerative lumbar disc disease. En Nachemson A (ed). Neck and back pain. The scientific evidence of causes, diagnosis and treatment. Philadelphia, Lippincott Williams \& Wilkins, 2000; pp.305-326.

75. Weinhardt, C., Heller, K.D., Weh, L.: Non-operative treatment of chronic lowback pain: specific back muscular strength training versus improvement of physical fitness. Z Orthop Ihre Grenzgeb 2001; 139: 490-495 (abstract).

Delgado-López, P.D.; Rodríguez-Salazar, A.; José Manuel Castilla-Díez, J.M.; Martín-Velasco, V.; Femández-Arconada, O.: Papel de la cirugía en la enfermedad degenerativa espinal. Análisis de revisiones sistemáticas sobre tratamientos quirúrgicos y conservadores desde el punto de vista de la medicina basada en la evidencia. Neurocirugía, 2005; 16: 142-157.

Correspondencia postal: Dr. P. Delgado López Servicio de Neurocirugía. Hospital General Yagüe. Avda. del Cid 96, 09005 Burgos. 